\title{
Phagocytosed Polyhedrin-Cytokine Cocrystal Nanoparticles Provide Sustained Secretion of Bioactive Cytokines from Macrophages
}

\author{
Astrid Wendler, Nicholas James, Michael H. Jones $(\mathbb{D}$, and Christian Pernstich
}

Cell Guidance Systems Ltd., Maia Building, Babraham Research Campus, Cambridge CB22 3AT, UK

Correspondence should be addressed to Michael H. Jones; michael.jones@cellgs.com

Received 2 March 2021; Accepted 26 April 2021; Published 15 May 2021

Copyright (C) 2021 Astrid Wendler et al. Exclusive Licensee Nanjing Agricultural University. Distributed under a Creative Commons Attribution License (CC BY 4.0).

\begin{abstract}
Many cells possess the ability to engulf and incorporate particles by phagocytosis. This active process is characteristic of microorganisms as well as higher order species. In mammals, monocytes, macrophages, and microglia are among the so-called professional phagocytes. In addition, cells such as fibroblast and chondrocytes are classified as nonprofessional phagocytes. Professional phagocytes play important roles in both the innate and adaptive immune responses, wound healing, and tissue homeostasis. Consequently, these cells are increasingly studied as targets and vectors of therapeutic intervention to treat a range of diseases. Professional phagocytes are notoriously difficult to transfect limiting their study and manipulation. Consequently, efforts have shifted towards the development of nanoparticles to deliver a cargo to phagocytic cells via phagocytosis. However, this approach carries significant technical challenges, particularly for protein cargos. We have focused on the development of nanoscale cocrystalline protein depots, known as $\mathrm{PODS}^{\circledR}$, that contain protein cargos, including cytokines. Here, we show that PODS are readily phagocytosed by nonprofessional as well as professional phagocytic cells and have attributes, such as highly sustained release of cargo, that suggest potential utility for the study and exploitation of phagocytic cells for drug delivery. Monocytes and macrophages that ingest PODS retain normal characteristics including a robust chemotactic response. Moreover, the PODS-cytokine cargo is secreted by the loaded cell at a level sufficient to modulate the behavior of surrounding nonphagocytic cells. The results presented here demonstrate the potential of PODS nanoparticles as a novel molecular tool for the study and manipulation of phagocytic cells and for the development of Trojan horse immunotherapy strategies to treat cancer and other diseases.
\end{abstract}

\section{Introduction}

Phagocytic cells engulf microscopic particles through an active process [1] and are classified as professional or nonprofessional with the latter taking up a more limited range of materials. Professional phagocytes include monocytes, macrophages, microglia, dendritic cells, and neutrophils. Nonprofessional phagocytes include chondrocytes, fibroblasts, erythrocytes, and epithelial cells.

Macrophages contribute to the innate and adaptive immune response. They also help to heal wounds and regulate tissue homeostasis [2]. Macrophages display phenotypic plasticity and can be polarized by microenvironmental cues to a spectrum of phenotypes very broadly characterized by the classically activated proinflammatory M1 phenotype and the alternatively activated anti-inflammatory M2 pheno- type. Macrophages are able to switch between these different phenotypes depending on changing requirements [3].

The role of macrophages in cancer underscores their importance. In precancerous lesions, macrophages target and remove defective cells, but as cancers develop, immuno-editing generates cancer cells that avoid this surveillance. Most tumor-associated macrophages (TAMs) are derived from monocytes which are constantly recruited into malignant tumors by chemotactic signals generated by the tumor, such as the CCL2 chemokine axis $[4,5]$. Once inside the cancer, the monocytes differentiate to macrophages. In later stages of disease, these macrophages are subverted by the cancer cells towards a M2-like phenotype, which actively supports the maintenance of cancer and suppresses the anticancer activity of other immune cells [6]. As a result of this central role, macrophages, and the proteins they secrete, are 
an important immunotherapeutic target for cancer drugs. Their tumor-infiltrating behavior also suggests their potential as vectors for targeted drug delivery.

The basic study of macrophages and the development of macrophage-based cellular therapies has been hampered by poor nucleic acid transfection efficiency [7]. This challenge has led many to explore the utility of nanoparticles carrying a cargo of functional molecules. To be useful, nanoparticles must be efficiently phagocytosed and release their cargo intact from the phagolysosome. The cargo must then be transported to targets within the cell or secreted by the cell to engage with extracellular targets.

A wide range of nanoparticles has been evaluated to date. These include drug-coated nanoparticles [8], radiosensitizers [9], drug-filled synthetic lipid particles [10], enzymes [11], and glucocorticoid prodrugs [12]. These nanoparticles have achieved limited success and a nanoparticle that can usefully deliver proteins, and particularly cytokines, to macrophages has yet to be developed.

Cytokines are key signaling molecules of the immune response. Recombinant cytokines such as IL-2, administered therapeutically, are able to activate immune cells and have been approved to treat certain metastatic cancers. Although high dose IL-2 therapy (Proleukin ${ }^{\circledR} /$ Aldesleukin) is highly effective in some patients, its acutely toxic side effects limit its use as a frontline therapy [13]. Effective cytokine nanoparticles containing IL-2 and other cargos may allow the development of a macrophage-mediated molecular Trojan horse strategy for the targeted delivery of cytokines to disseminated cancer.

PODS are nanoscale $(200 \mathrm{~nm}-5 \mu \mathrm{m})$ protein cocrystals built from the polyhedrin protein. Their distinctive cubic structure is determined by intracellular assembly of a specific polyhedrin protein derived from the Bombyx mori cypovirus [14]. A cargo protein can be incorporated into PODS during crystal assembly via an immobilization tag, typically using a fragment of the polyhedrin $\mathrm{H} 1$ finger. PODS happen to be the ideal size, shape, and rigidity for phagocytic uptake [15]. PODS are also nonbrittle and temperature-stable. In the presence of proteases, PODS slowly degrade and typically release their cargo over a period of one-two months. Fortuitously, this degradation results in the release of cargo protein that retains bioactivity.

Here, we explore the potential of PODS for delivering functional cytokines to monocytes and macrophages. We show that PODS particles are very efficiently phagocytosed and that cargo cytokines either evade or withstand the harsh conditions of the phagolysosome allowing secretion by macrophages. Moreover, secretion levels achieved are sufficient to produce distinct phenotypic changes in heterogenous cocultured cells. Our findings demonstrate the utility of PODS for macrophage-based research and suggest their potential utility in Trojan horse drug delivery strategies to treat cancer and other diseases.

\section{Results}

2.1. Efficient Phagocytosis of PODS Crystals into Macrophages. PODS protein crystals form near-perfect, transparent cubes that are generated in insect cells. The crystal size varies depending on the number of crystals in an individual cell, the size of cell, and the amount of time elapsed from their initial formation. The sizes of crystals vary between $200 \mathrm{~nm}$ and $5 \mu \mathrm{m}$, with most $0.5-2.0 \mu \mathrm{m}$. The crystals' angular shape and overall appearance make them distinct from cells and thus easily identifiable even at low magnification (Supplementary Figure 1). We first tested our expectation that PODS crystals can be phagocytosed by primary murine monocytes and macrophages. Murine bone marrow-derived monocytes (BMDM) were isolated according to Wagner et al. [16] from the tibias of C57BL/6 mice. Cells were cultured for 5 days before they were incubated with fresh complete medium containing PODS M-CSF or PODS GM-CSF at a ratio of 5 crystals per cell. PODS crystals were phagocytosed into the BMDMs with high efficiency (Figure 1(a)), seemingly without negatively influencing their behavior (supplementary material, video 1). Moreover, when PODS GM-CSF-loaded BMDMs were incubated with a further 5 PODS per cell, they ingested all the additional PODS crystals (Supplementary Figure 2) while maintaining regular motility and morphological changes, such as protruding leading edges and uropods (Supplementary material, video 2). As far as we could tell, all crystals were taken up by the cells equally effectively regardless of size.

Following successful phagocytosis into primary phagocytes, we used THP-1 cells as an in vitro model for macrophages, based on Genin et al's study [17]. These cells can be readily polarized to different macrophage types. Figure 1(b) shows a schematic of the workflow. THP-1 monocytes were differentiated into M0 macrophages by incubating cells in complete medium supplemented with phorbol-12-myristate-13-acetate (PMA). M0 cells were then polarized to M1 or M2 type macrophages by incubating cells in complete medium supplemented with either IFN- $\gamma$ and LPS or IL-4 and IL-13, respectively. Once polarized, the medium was replaced with fresh complete medium containing PODS Empty crystals (polyhedrin-only, containing no cargo protein) in a ratio of 10 crystals per cell, and uptake of PODS was monitored repeatedly over $24 \mathrm{~h}$ using a live cell imaging system. Brightfield images taken after $24 \mathrm{~h}$ show that almost all crystals have been taken up by the cells, independent of their polarization status (Figure 1(c) and Supplementary Figure 3). Moreover, nonprofessional phagocytes, such as chondrocytes, NIH-3T3 fibroblasts, and $\mathrm{C} 2 \mathrm{C} 12$ myoblasts, were equally capable of ingesting PODS efficiently (Supplementary Figure 4).

2.2. Viability and Polarization Status of PODS-Loaded Macrophages. Given the cytotoxic effect generated by different types of nanoparticles on macrophages in previous studies [18-20], the viability of M0, M1, and M2 cells was examined at $48 \mathrm{~h}$ (Figure 2(a)) and 96h (Supplementary Figure 5) after uptake of different numbers of PODS crystals. Results from WST-8 assays demonstrated that cell viability was not reduced compared with untreated cells (M0, M1, and M2 cells which were not loaded with PODS), suggesting that the PODS particles were nontoxic at a 

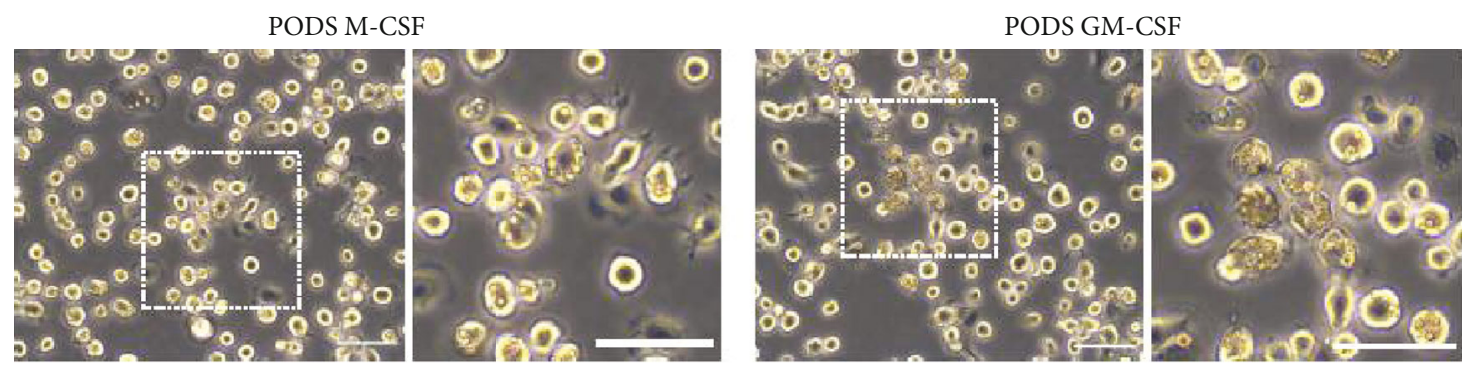

(a)
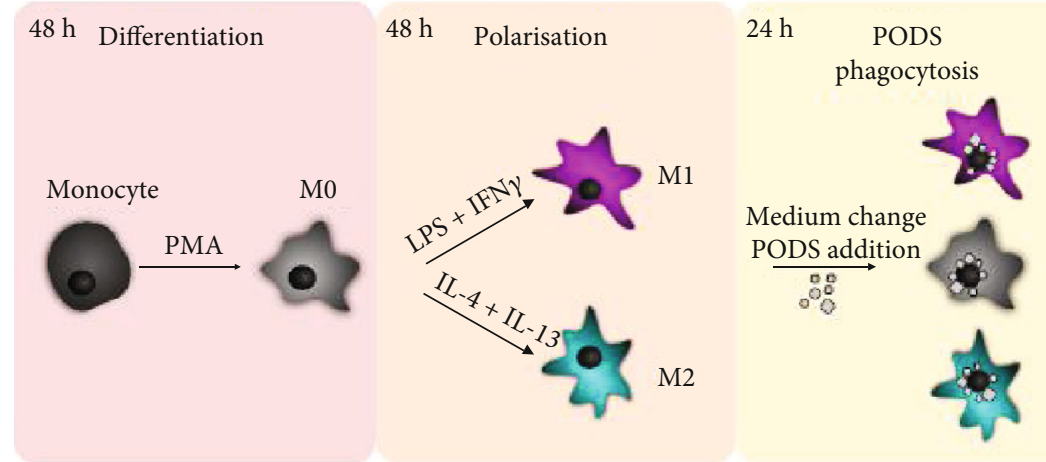

(b)
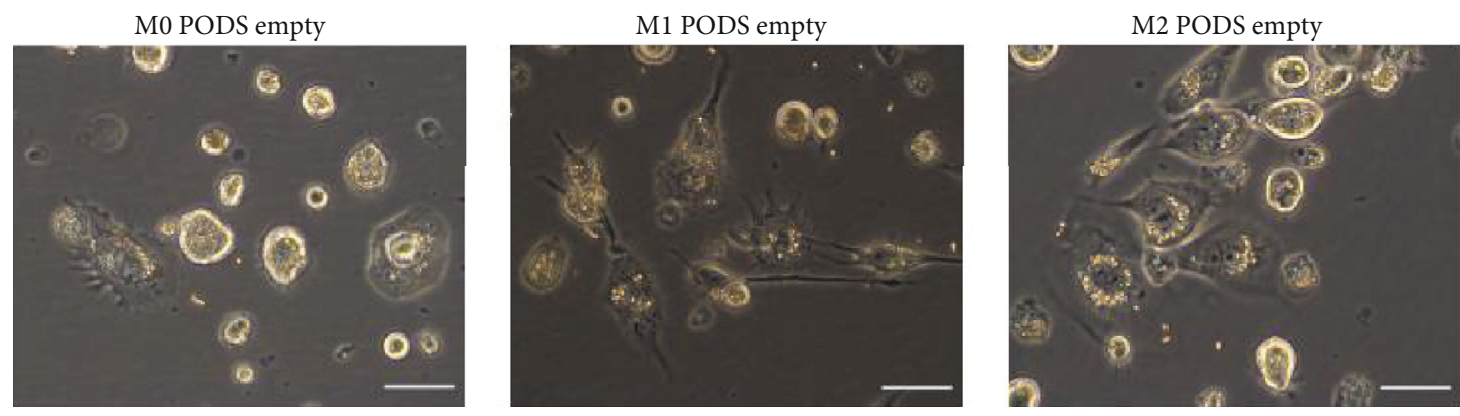

(c)

FIGURE 1: Phagocytosis of PODS protein crystals into professional phagocytes. PODS Empty, PODS M-CSF, and GM-CSF represent PODS containing no cargo protein, M-CSF, and GM-CSF, respectively. GM-CSF: granulocyte-macrophage colony-stimulating factor; M-CSF: macrophage colony-stimulating factor. (a) Brightfield images of PODS M-CSF and GM-CSF loaded bone marrow-derived mouse macrophages at a magnification of $40 \mathrm{x}$ with a scale bar of $100 \mu \mathrm{m}$. The dashed square marks the area of the zoom shown in the right image of each panel. (b) Schematic overview of the THP-1 differentiation (M0), polarization (M1, M2), and PODS phagocytosis workflow. (c) THP-1 cells were differentiated first into M0 macrophages and then further polarized into either M1 or M2 macrophages according to the workflow depicted in (b). Cells of the various differentiated types were cultured with PODS Empty at a concentration of 10 PODS per cell for $24 \mathrm{~h}$ and subsequently imaged at a magnification of 40x. The scale bar is $100 \mu \mathrm{m}$.

median dosage range of up to 15 PODS/cell. Neither PODS Empty nor PODS FGF-10 (a growth factor which alone is nontoxic to macrophages) [21] had any negative effect on macrophage viability. However, there was a limit to how many PODS a macrophage could ingest before going into apoptosis. Ingestion of around 50 PODS per cell clearly leads to some cells "bursting" into apoptotic bodies (Supplementary figure 6).

The activation and polarization of monocytes towards an M1-like phenotype upon contact with a foreign body are a key element of the innate immune system. To determine if uptake of PODS crystals by monocytes and macrophages influences their polarization status, we measured the secretion of IL-6, a marker for M1 polarization, before and after
PODS uptake. First, we confirmed that IL-6 can indeed be used as a proxy for polarization status, showing that M1 cells raised concentration levels of IL- 6 in the cell culture media to more than $4 \mathrm{ng} / \mathrm{ml}$ directly after polarization (Figure 2(b)), whereas neither nonactivated THP-1 cells nor M0 and M2 cells raised IL-6 concentration above background level.

The polarization medium was changed to standard media with no growth factors. The cells were then divided into two groups. In the first group, PODS FGF-2 were added and phagocytic uptake was allowed to proceed for $24 \mathrm{~h}$ to load the cells. Following this incubation period, both loaded and unloaded M1 cells secreted IL-6 further, up to concentrations of $200 \mathrm{pg} / \mathrm{ml}$ (Figure 2(c)). The continuous secretion of IL-6 from PODS-loaded M1 cells at the same level as unloaded 


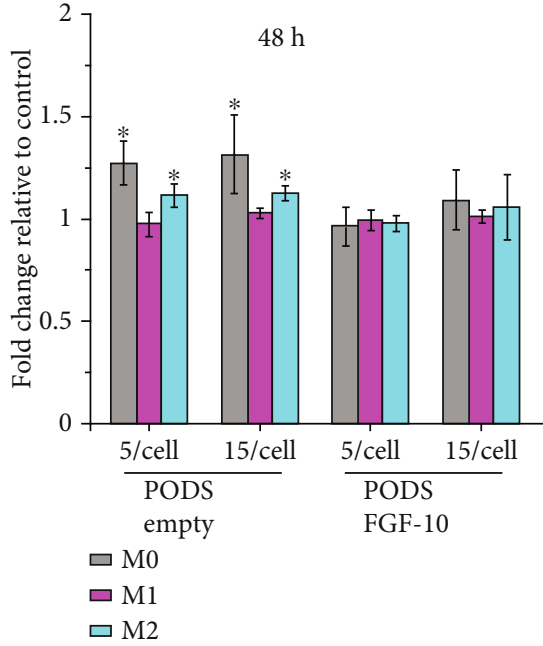

(a)

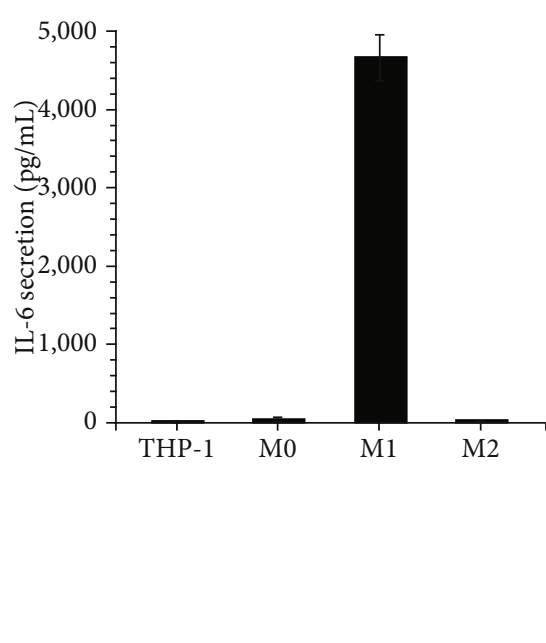

(b)

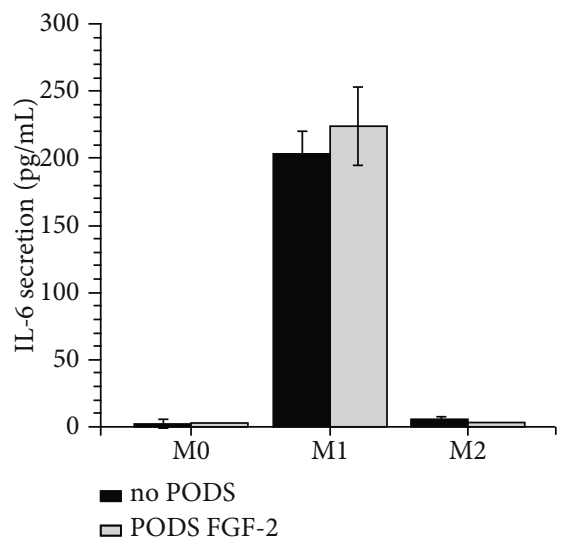

(c)

FIGURE 2: Characterization of professional phagocyte postphagocytosis of PODS proteins. (a) M0, M1, and M2 macrophages were incubated with PODS Empty or PODS FGF-10 (1:5 and 1:15) for $24 \mathrm{~h}$ in a 96-well TC plate. Subsequently, the medium was changed and viability of cells was measured $48 \mathrm{~h}$ after PODS uptake using a colorimetric assay (Orangu ${ }^{\mathrm{TM}}$, Cell Guidance Systems). The fold change in viability was calculated relative to unloaded macrophages of the same polarization state. Error bars represent the standard deviation of samples $(n=3)$. *Significant differences to control $(p<0.05)$. (b) Medium of THP-1, M0, M1, and M2 was collected directly after polarization and tested for the presence of IL- 6 by ELISA. Error bars represent the standard deviation of samples $(n=3)$. (c) The same cells were then washed and incubated with PODS FGF-2 for $24 \mathrm{~h}$ in a fresh full growth medium. The medium was then collected and analyzed for the presence of IL- 6 by ELISA. Error bars represent the standard deviation of samples $(n=3)$. There was no significant difference between samples and controls.

M1 cells suggests that the uptake of PODS crystals did not alter their polarization status. Equally, the uptake of PODS crystals did not induce IL-6 secretion of M0 and M2 cells, suggesting that phagocytosis of PODS crystals by itself does not change the polarization status of any macrophage type.

\subsection{PODS-Loaded Macrophages Retain Functionality. Hav-} ing established that PODS particles are efficiently taken up and do not affect macrophage viability, we assessed the cells for their characteristic ability to migrate and follow chemotactic signals.

To quantify potential changes in mobility due to uptake of PODS, M0 cells were incubated with PODS IL-2 for $24 \mathrm{~h}$ and then observed with a live cell imaging system under normal growth conditions for a further $24 \mathrm{~h}$. Movement of unloaded and PODS IL-2-loaded cells was analyzed by tracking each of 25 cells across 720 frames taken at 2-minute intervals (Figure 3(a)). When comparing tracks for both the distance migrated and randomness, there was no detectable difference in the mobility of cells with and without PODS.

To test the ability of PODS IL-2-loaded M0 cells to follow a chemotactic gradient, the above experiment was repeated with a slight variation: after PODS uptake, cells were taken into serum-free medium and transferred into the observation area of chemotactic slides ( $\mu$-Slide, Ibidi) in between two reservoirs. Reservoir 1 was filled with medium containing $10 \%$ serum, acting as a chemoattractant, and reservoir 2 was filled with serum-free medium. The movement of unloaded and PODS IL-2-loaded cells was investigated by tracking 26 cells for $12 \mathrm{~h}$ (1 frame per minute) each as described above (Figure 3(b)). The analysis of this tracking data revealed that uptake of PODS crystals does not impair migration of macrophages towards a chemoattractant as both unloaded and PODS-loaded M0 cells followed the chemical cue to the same extent.

Another characteristic of macrophages is their ability to traverse narrow capillaries and to extravasate into surrounding tissues. To assess if PODS-loaded macrophages retain the ability to traverse narrow spaces, PODS eGFP- (enhanced green fluorescent protein-) loaded M0 cells were placed into a serum-free medium and transferred to 24 -well tissue culture inserts containing extended $8 \mu \mathrm{m}$ diameter pores, akin to the narrowest blood capillaries, in their bases. Maintenance medium containing $10 \%$ serum was used as a chemoattractant in the lower well (Figure 4(a)). After $24 \mathrm{~h}$ of incubation, the number of cells that migrated towards serum was compared to the number of cells in a second chamber which lacked serum as a chemoattractant. Visibly larger cell numbers migrated into the wells with chemoattractant demonstrating that loaded M0 cells were capable of passing through the narrow pores and actively migrated through the $8 \mu \mathrm{m}$ pores in the presence of a chemoattractant.

We are interested in the potential of PODS-loaded macrophages to provide a delivery mechanism to modulate the tumor microenvironment. Macrophages are actively recruited by chemoattractants, such as CCL2, secreted by solid tumors [6]. To explore whether PODS-loaded macrophages responded to chemotactic cues secreted by cancer cells, we use conditioned medium from a cancer cell line to attract PODS eGFP-loaded macrophages. The same cell chamber described above was used with the PODS-loaded cells in the insert and the conditioned media below. To 

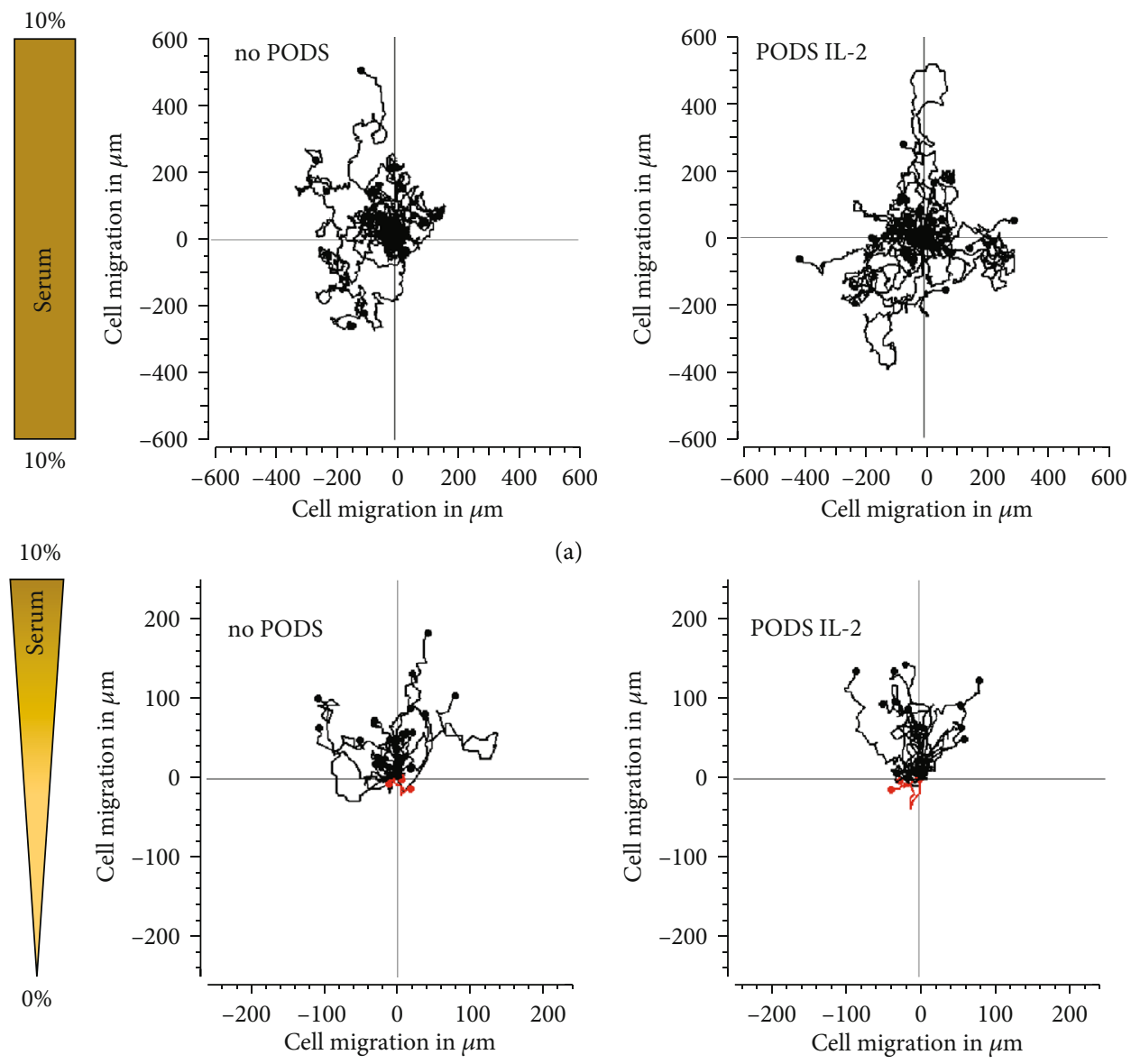

(b)

FIgURe 3: Mobility and chemotaxis of M0 cells loaded up with PODS. (a) Mobility of PODS IL-2 loaded macrophages. The mobility of empty and PODS IL-2-loaded macrophages (10 PODS/cell) in a full growth medium was monitored using a live cell imaging system. Images were taken every $2 \mathrm{~min}$ for $24 \mathrm{~h}$ and analyzed using the manual tracker of ImageJ and the chemotaxis and migration tool (Ibidi). 25 cells per condition were followed, and their migration tracks were visualized as a rose plot, in which each line represents the track of an individual cell over 24 hours. Track positions were normalized so that each track starts at the $(0,0)$ coordinate. (b) Empty and PODS IL-2-loaded macrophages were transferred into Chemotaxis $\mu$-Slides (Ibidi) and tested for their ability to follow a chemoattractant gradient generated from 0 to $10 \%$ BCS. Images were taken every $1 \mathrm{~min}$ for $12 \mathrm{~h}$ and analyzed using the manual tracker utility of ImageJ and the chemotaxis and migration tool (Ibidi). 26 cells per condition were followed, and their migration tracks were visualized as a rose plot, in which each line represents the track of an individual cell over 12 hours. Track positions were normalized so that each track starts at the $(0,0)$ coordinate. Tracks of cells migrated towards the $10 \%$ BCS source are shown in black (up), whereas tracks of cells migrated away from the BCS source are shown in red (down).

condition the media, A375 cells (a human malignant melanoma cell line) were grown for 3 days in $10 \%$ serum containing growth medium. The conditioned medium was then used as a chemoattractant in the bottom well (Figure 4(b)) with the PODS-loaded macrophages placed in the top chamber in media containing $10 \%$ serum. As a control, a second cell chamber was set up without conditioned media, containing the same $10 \%$ serum in the lower well and upper insert. The cells were incubated for $24 \mathrm{~h}$, and then phase contrast as well as fluorescent microscopy was used to observe the number of cells that had moved into the well from the insert. This experiment clearly showed that PODS eGFP-loaded M0 cells were attracted specifically by the cancer cell lineconditioned medium, migrating through the $8 \mu \mathrm{m}$ pores towards the chemotactic source.
2.4. Release of Cargo from PODS-Loaded Macrophages. Macrophages orchestrate immune responses by secreting a range of cytokines and other signaling molecules which affect other immune cells. We wished to determine if it is possible to modulate this secretion profile using PODS. To test if ingested PODS cargo protein is released from the macrophages into the medium, M0 cells were incubated with PODS IL-6 for $24 \mathrm{~h}$, washed twice, and then incubated for a further 4 days in normal growth medium containing no additional PODS. Medium conditioned by the loaded macrophages was collected and analyzed for the presence of IL- 6 by ELISA (Figure 5(a)). Against expectations, cargo protein IL-6 was readily detected in the cell culture medium. Moreover, the levels of IL- 6 were dose-dependent: the more PODS IL- 6 were loaded into M0 cells, the more IL- 6 could be detected 

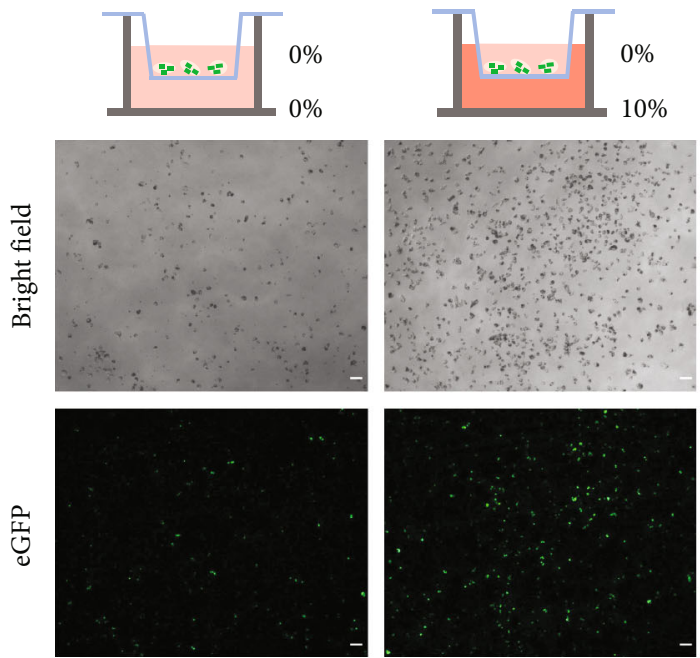

(a)
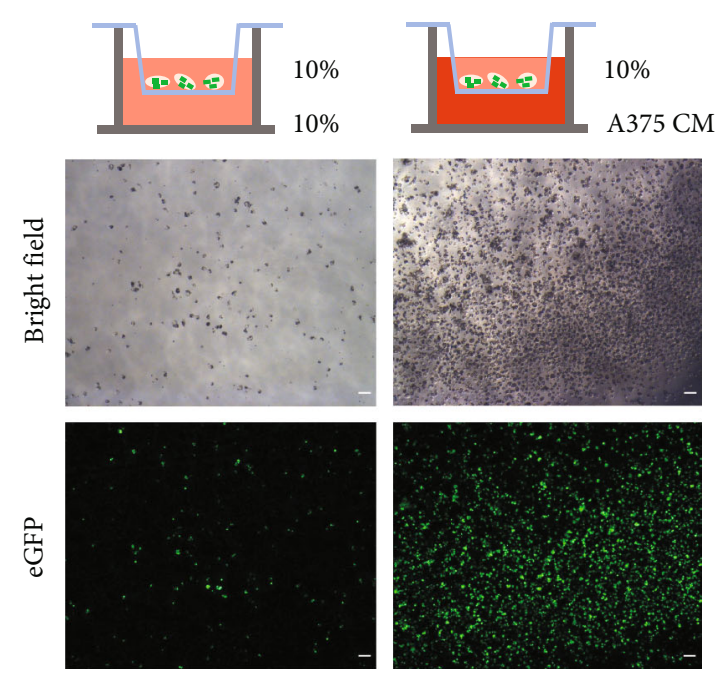

(b)

FIGURE 4: Directed migration of PODS eGFP loaded macrophages through $8 \mu \mathrm{m}$ pores. (a) PODS eGFP-loaded macrophages (5 PODS/cell) in a serum-free medium were transferred into 24 -well inserts with $8 \mu \mathrm{m}$ pores. The bottom well was filled with either RPMI-1640 only (left) or RPMI-1640 supplemented with 10\% FBS (right). The top images show to the bottom well-migrated cells in the brightfield channel, and the lower images show the corresponding fluorescent channel after $24 \mathrm{~h}$ of incubation. (b) PODS eGFP-loaded macrophages follow chemical signals from the melanoma cell line through $8 \mu \mathrm{m}$ pores. PODS eGFP-loaded macrophages (5 PODS/cell) in full growth medium were transferred into 24 -well inserts with $8 \mu \mathrm{m}$ pores. The well was filled with either full growth medium (left) or medium that was conditioned with A375 cells for 3 days (right). The top images show the bottom well-migrated cells in the brightfield channel, and the lower images show the corresponding fluorescent channel after $24 \mathrm{~h}$ of incubation. The cells were imaged at a magnification of $10 \mathrm{x}$, and the scale bar is $100 \mu \mathrm{m}$.

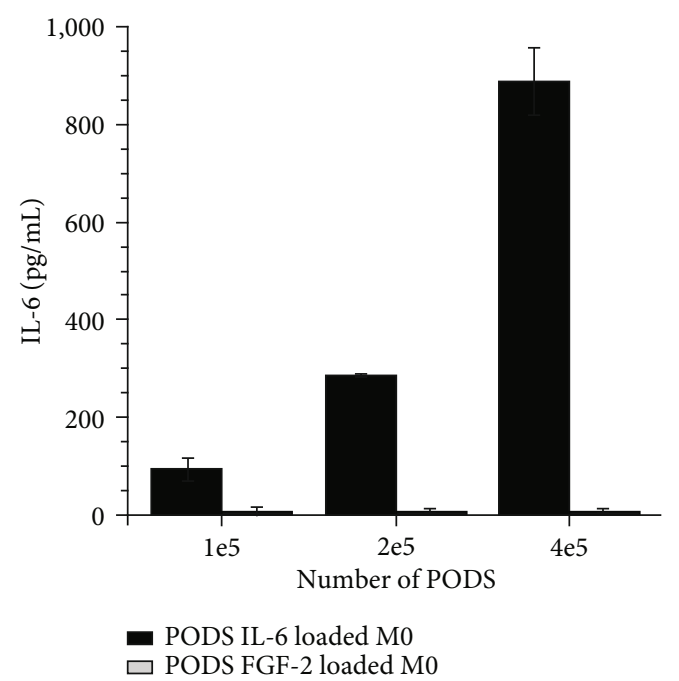

(a)

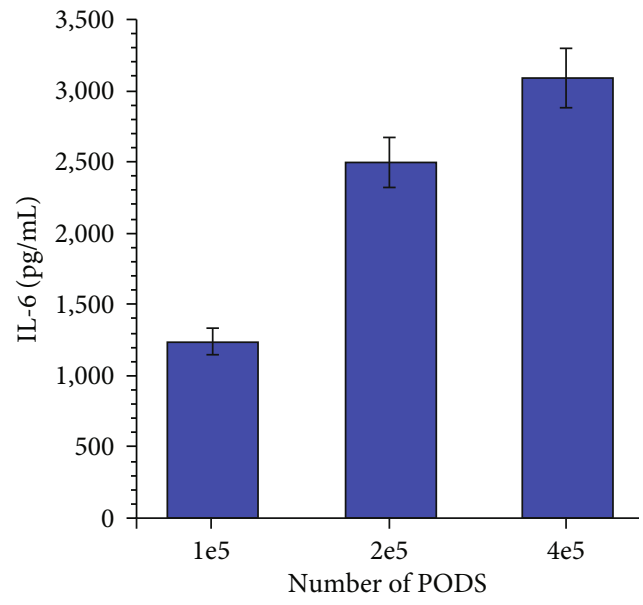

(b)

FIgURE 5: Release of IL-6 from PODS IL-6 loaded macrophages. (a) M0 was loaded with 5, 10, or 20 of either PODS IL-6 or PODS FGF-2 per cell (equivalent to $1 \times 10^{5}, 2 \times 10^{5}$, and $4 \times 10^{5}$ PODS per 96-well). Cells were washed and incubated in full growth medium for $4 \mathrm{~d}$. The medium was collected and tested for the presence of IL- 6 in the medium. Error bars represent the standard deviation of samples $(n=3)$. IL-6 levels of controls were below background. (b) The same amounts of PODS IL-6 as before were spun down to the bottom of a 96-well plate and incubated for $4 \mathrm{~d}$ in full growth medium. The medium was collected, and levels of IL-6 were measured by ELISA. Error bars represent the standard deviation of samples $(n=3)$.

in the medium after 4 days. As a control, we also measured the amount of IL-6 released from similar numbers of nonphagocytosed PODS IL-6 (Figure 5(b)). The amount of IL-6 released from naked PODS was 3-10 times higher than that from PODS taken up by macrophages suggesting either reduced rates of cytokine release from macrophages or increased rates of degradation. As a further control, M0 cells were incubated with PODS FGF-2 in the same way and levels 
of IL- 6 in the medium were analyzed. Levels of IL- 6 in the medium were shown to remain below background $(<10 \mathrm{pg} / \mathrm{ml})$ (Figure 5(a)).

\subsection{Cargo Released from PODS-Loaded Macrophages Effects} a Phenotypic Change in Heterogenous Cocultured Cells. After demonstrating secretion of cytokine cargo by macrophages, we wished to determine if release rates are sufficient to drive changes in phenotype in cocultured heterogenous cells. Here, a proliferation assay using NIH-3T3 cells that are responsive to the FGF-2 protein was performed. NIH-3T3 cells were seeded in normal growth medium into wells of a 24-well plate. After $24 \mathrm{~h}$, the media was replaced with serum-free medium. Separately, M0, M1, and M2 polarized macrophages were incubated with PODS FGF-2 (10 PODS/cell) for $24 \mathrm{~h}$ to facilitate phagocytosis. PODS FGF-2-loaded macrophages were then detached, changed into serum-free medium, and seeded into 24 -well TC inserts which were subsequently placed into the wells that contained NIH-3T3 cells. Cocultures were incubated for a further 4 days, and the number of NIH-3T3 cells was assessed by performing an Orangu assay (Figure 6). Coincubation of all types of PODS FGF-2-loaded macrophages supported the growth of FGF2-responsive NIH-3T3 cells compared to unloaded macrophages.

\section{Material and Methods}

3.1. PODS Crystal Synthesis. All PODS proteins were synthesized as previously described $[22,23]$. All constructs were fused to the H1 immobilization tag [24]. Briefly, baculovirus (BV) DNA and transfer DNA were cotransfected into standard Spodoptera frugiperda 9 (Sf9) cells using TransITInsect (Mirus Bio). Resulting infective BV was harvested, and a plaque purification was then performed to isolate a single recombinant $\mathrm{BV}$. Isolated plaques were first screened, and positive BV was then harvested, expanded, and finally used to infect large scale Sf9 cell cultures to produce PODS crystals. Subsequently, crystals were harvested and purified by lysing Sf9 cells using successive rounds of sonication and PBS washes. Finally, purified PODS were sterility tested and lyophilized prior to use in experiments. Although equivalence depends on context, $1.5 \times 10^{4}$ PODS crystals are approximately functionally equivalent to $1 \mathrm{ng}$ of many standard growth factors, cytokines, or chemokines in terms of bioactivity [23].

3.2. Cell Culture and Differentiation. Monocyte suspension cells (THP-1, Public Health England Culture Collection) were cultured undifferentiated in RPMI-1640 (A10491, Gibco) supplemented with 10\% BCS (30-2030, ATCC) (complete medium). For M0 differentiation, THP-1 cells were centrifuged and the conditioned media were replaced with fresh complete medium supplemented with $100 \mathrm{ng} / \mathrm{ml}$ phorbol 12myristate-13-acetate (PMA, Sigma P8139). After 48 h, M0 macrophages were further differentiated into M1 or M2 macrophages as described [17, 25]. Briefly, M0 differentiation medium was removed and adherent M0 cells were washed twice with serum-free medium. For further differentiation,

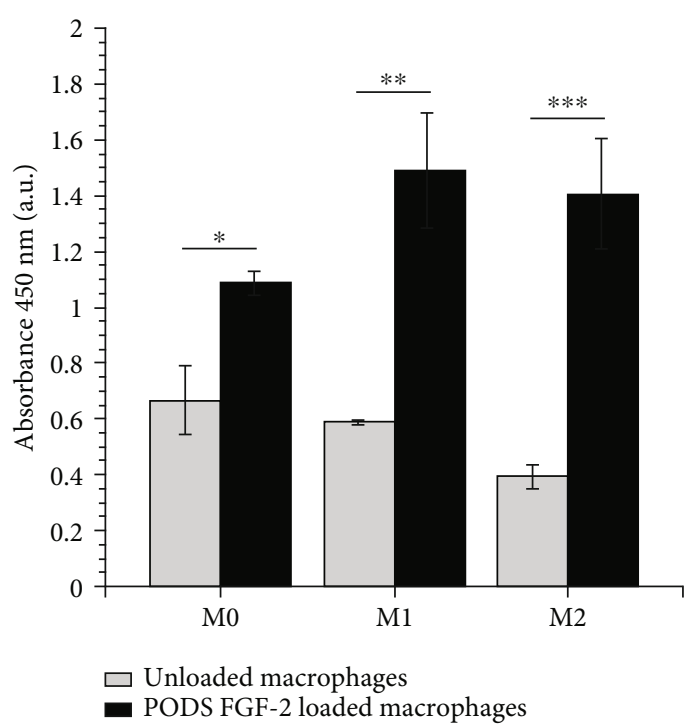

FIgURE 6: Released cargo from phagocytosed PODS is bioactive. Bioactivity of FGF-2 released from PODS FGF-2-loaded macrophages. Unloaded and FGF-2-loaded macrophages (M0, $\mathrm{M} 1$, and M2 with $10 \mathrm{PODS} /$ cell) in TC inserts were incubated with FGF-2-reactive NIH-3T3 cells under serum-free conditions for $4 \mathrm{~d}$. Proliferation of NIH-3T3 cells was measured using a colorimetric assay (Orangu ${ }^{\mathrm{TM}}$, Cell Guidance Systems). Error bars represent the standard deviation of samples $(n=2)$. Significant differences to control $\left({ }^{*} p<0.05,{ }^{* *} p<0.01\right.$, and $\left.{ }^{* * *} p<0.001\right)$.

complete medium supplemented with either $100 \mathrm{ng} / \mathrm{ml}$ LPS and $20 \mathrm{ng} / \mathrm{ml} \mathrm{IFN-} \gamma$ (M1) or $20 \mathrm{ng} / \mathrm{ml} \mathrm{IL}-4$ and $20 \mathrm{ng} / \mathrm{ml}$ IL-13 (M2) was added to adherent M0 cells and then incubated for up to a further $48 \mathrm{~h}$. A375 cells (ATCC) were cultured in DMEM (41966, Gibco) supplemented with $10 \%$ FBS (F7524, SIGMA). Cells were seeded at a density of $2 e 4$ cell $/ \mathrm{cm}^{2}$ and passaged every 3 days. TF- 1 cells (ATCC) were cultured in RPMI- 1640 supplemented with $2 \mathrm{ng} / \mathrm{ml} \mathrm{GM-CSF}$ and $10 \%$ FBS. Cells were seeded at a density of $2 e 5$ cells $/ \mathrm{ml}$ and passaged twice a week.

3.3. Phagocytosis. PODS crystals were either centrifuged onto tissue culture (TC) plates, or centrifuged and dried onto TC plates, or premixed with phagocytic cells for up to 48 hours before plating. Phagocytic cells were then seeded where necessary and subsequently cultured in complete medium. Phagocytosis into cells was demonstrated by monitoring with a real-time cell history recorder (JuLi stage, NanoEnTek Inc.).

3.4. Viability Assay. To test macrophage viability after PODS uptake, THP- 1 cells were seeded at a density of $2 e 5$ cells $/ \mathrm{ml}$ and differentiated and polarized as described above. $1 e 6$ or $3 e 6$ PODS Empty and PODS FGF-10 per $\mathrm{ml}$ were added to unpolarized M0 and polarized M1 and M2 macrophages, resulting in an average of 5 or 15 PODS/cell, respectively. Cells were then incubated for $48 \mathrm{~h}$ and $96 \mathrm{~h}$, and a WST-8 assay (Orangu ${ }^{\mathrm{TM}}$, Cell Guidance Systems) was performed to determine cell viability. 
3.5. Mobility Tracking. THP-1 cells were seeded at a density of $2 e 5$ cells $/ \mathrm{ml}$ in 24-well plates and differentiated into M0 macrophages as described above. M0 cells were then incubated with PODS IL- 2 for $24 \mathrm{~h}$ in complete medium. The medium was then renewed, and cells were recorded using a real-time cell history recorder (JuLi stage, NanoEnTek Inc.). Images were taken every $2 \mathrm{~min}$ for $24 \mathrm{~h}$ and then analyzed using the manual tracker of ImageJ and the chemotaxis and migration tool (Ibidi).

3.6. Chemotaxis. THP-1 cells were seeded at a density of $2 e 5$ cells $/ \mathrm{ml}$ in T25 TC flasks and differentiated into M0 macrophages as described above. M0 cells were then incubated with PODS IL-2 for $24 \mathrm{~h}$ in complete medium. Subsequently, M0 macrophages were washed with PBS and then detached using an enzyme-free detachment buffer (Cell Dissociation Buffer, Gibco). Cells were then centrifuged, recounted, and seeded in serum-free RPMI-1640 medium at a density of $2 e 6$ cells $/ \mathrm{ml}$ into the observation area of Chemotaxis $\mu$-Slides (Ibidi). 10\% FBS was used as a chemoattractant. Cells in the observation area were recorded using a real-time cell history recorder (JuLi stage, NanoEnTek Inc.). Images were taken once per minute for $12 \mathrm{~h}$ and then analyzed using the manual tracker of ImageJ and the chemotaxis and migration tool (Ibidi).

3.7. Directed Migration. THP-1 cells were seeded at a density of $2 e 5$ cells $/ \mathrm{ml}$ in T25 TC flasks and differentiated into M0 macrophages as described above. M0 cells were then incubated with $5 e 5$ PODS eGFP per $\mathrm{mL}$ for $24 \mathrm{~h}$ in complete medium (2.5 PODS/cell). Subsequently, M0 macrophages were washed with PBS and then detached using an enzyme free detachment buffer (Cell Dissociation Buffer, Gibco). Cells were centrifuged, recounted, and seeded in serum-free or 10\% FBS containing RPMI-1640 medium at a density of $1 e 6$ cells/ml into 24 -well inserts. The bottom well was then filled with either serum-free medium, 10\% FBS-containing medium, or 3-day conditioned medium of A375 cells. Microscope images of the bottom well and the insert were taken after $24 \mathrm{~h}$ of migration.

3.8. Endogenous IL-6 Secretion. THP-1 cells were seeded at a density of $2 \mathrm{e} 5 \mathrm{cells} / \mathrm{ml}$ in 24 -well plates and differentiated and polarized as described above. The conditioned polarization medium was then collected and stored at $-20^{\circ} \mathrm{C}$ for later analysis. Macrophages were then incubated with $2 e 6$ PODS FGF-2 (or $2 e 6$ PODS Empty) per $\mathrm{ml}$ for $24 \mathrm{~h}$ in fresh complete medium (10 PODS/cell). The medium was then collected and stored at $-20^{\circ} \mathrm{C}$. Medium samples were tested for the presence of IL- 6 by ELISA (DY206, R\&D systems) according to the manufacturer's protocol.

3.9. Release of IL-6 from PODS IL-6-Loaded Macrophages. THP- 1 cells were seeded at a density of $2 e 5$ cells $/ \mathrm{ml}$ in $96-$ well plates and differentiated into M0 macrophages. M0 cells were then incubated with $1 e 6,2 e 6$, or $3 e 6$ PODS IL- 6 per ml for $24 \mathrm{~h}$ in complete medium (resulting in 5, 10, or 30 PODS/cell). Cells were then washed twice with PBS, and fresh complete medium was added and cells were incubated for 4 days. Additionally, the same number of naked PODS ${ }^{\circledR}$
IL-6 was added to the wells of a 96-well plate and was spun down at $3000 \mathrm{x}$ g for $25 \mathrm{~min}$. The PBS was removed, and the plate was dried in a laminar flow hood and then supplemented with full growth medium for $4 \mathrm{~d}$. After incubation, the medium was collected and subsequently tested by IL-6 ELISA (DY206, R\&D systems) according to the manufacturer's protocol.

3.10. Functional Assay. THP-1 cells were seeded at a density of $2 e 5$ cells $/ \mathrm{ml}$ in 6-well plates and differentiated and polarized as described above. Macrophages were then incubated with $2 e 6$ PODS FGF-2 per $\mathrm{ml}$ for $24 \mathrm{~h}$ in complete medium (10 PODS/cell). Subsequently, macrophages were washed with PBS and then detached using an enzyme-free detachment buffer (Cell Dissociation Buffer, Gibco). Cells were then centrifuged, recounted, and seeded in serum-free TF-1 conditioned medium at a density of $2 e 6$ cells $/ \mathrm{ml}$ into 24 -well inserts. TF-1 cells were seeded at a density of $3 e 4$ cells $/ \mathrm{cm}^{2}$ in full growth medium into a 24-well plate. After one day of growth, the medium was changed to RPMI-1640 supplemented with $0.5 \%$ BCS and the macrophage containing inserts were added. Cells were coincubated for 4 days, and the viability of the TF-1 cells was measured performing a WST-8 assay (Orangu ${ }^{\mathrm{TM}}$, Cell Guidance Systems).

3.11. Derivation of Primary Monocytes from Mouse Tibia. Murine bone marrow-derived monocytes were isolated according to Wagner et al. [16]. Briefly, the tibias of 3 C57BL/6 mice were prepared for harvest. After washing once in $96 \%$ ethanol and twice in PBS, the distal end of each bone was cut with fine scissors and the bones were flushed with warm medium (M199 supplemented with 10\% FBS and 1\% Penicillin/Streptomycin) using a $28-\mathrm{G}$ needle and a $1 \mathrm{ml}$ syringe. The flow-through from the bones was collected and filtered through a $70 \mu \mathrm{m}$ cell strainer. The cell suspension was centrifuged at $200 \mathrm{xg}$ for $10 \mathrm{~min}$ at RT. The pellet was washed with $25 \mathrm{ml}$ of medium and centrifuged again. The cells were then seeded at $0.5 e 6 \mathrm{cells} / \mathrm{ml}$ in 6-well ultra-lowattachment plates. Cells were cultured for 5 days before using in experiments.

\section{Discussion}

Here, we have explored the potential utility of PODS protein crystal nanoparticles as vectors for modifying the cytokine secretion profile of phagocytic cells. We first demonstrated that PODS are readily, consistently, and efficiently taken up by professional phagocytes including murine bone marrowderived monocytes and THP-1 cell line-derived macrophages regardless of polarization states.

PODS are taken up by a variety of cells remarkably well and may have optimal characteristics for phagocytosis. Studies $[26,27]$ focusing on shape-dependent differences of uptake with minor size variation and phagocytosis as a 2stage process, respectively, could detect pronounced variations albeit measured in minutes to dozens of minutes. While the exact mechanisms of phagocytosis are still unclear, feasibly, these could differ depending on particle size and indeed phagocytic cell type, yet on the timescales utilised in our 
studies, differences could not be observed. Further experiments are needed, e.g., loss of function experiments, to elucidate more detail about the exact process of how PODS crystals are phagocytosed.

A potential problem with protein nanoparticle loading into macrophages is the destruction of the particle and any cargo by the phagolysosome due to its low $\mathrm{pH}$ and lysosomal proteases. PODS are particularly stable in acidic environments and protect their cargo due to their tightly packaged format [28]. Following phagocytosis, we have demonstrated sustained release from macrophages of a cytokine encapsulated into PODS crystals. The released cytokine could be detected reliably, and in a dose-dependent manner, in macrophage culture medium. This was observed with macrophages averaging as few as 5 PODS per macrophage. Even more importantly, released protein was shown to be bioactive as verified by proliferation of an FGF- 2 reactive cell line upon coculture with PODS FGF-2-loaded macrophages.

It is not clear how PODS crystals are able to withstand the internal machinery of macrophages and secrete their cargo intact. One possibility is that the unique structure of PODS allows them to endure, or simply overwhelm, the hostile phagolysosome environment. However, work by others suggests an alternative mechanism. Detection of active cargo released from macrophages was also demonstrated for macrophages loaded with antiretroviral nanoparticles [29] which avoided intracellular degradation and were recycled to the plasma membrane. The perinuclear localization of PODS crystals after uptake that we observed for PODS crystals would support such a mechanism.

Importantly, the uptake of PODS does not seem to be cytotoxic nor does it fundamentally change characteristics of professional phagocytes such as mobility or chemotaxis. This lack of cytotoxicity is seen even when multiple PODS crystals are loaded into a single macrophage swelling the cell's size. Maintenance of functionality is an important prerequisite for most useful applications of any macrophage nanoparticles.

Selective targeting of drugs to diseased tissue, notably cancer, is urgently needed to reduce levels of off-target toxicity and to increase efficacy. The use of cells as a therapeutic delivery system has been under discussion since the 1970 s [30], and the use of macrophages for a Trojan horse drug delivery strategy to treat cancer was proposed in the 1990s [31]. Macrophages are particularly attractive for cancer therapy because of their tumor-infiltrating behavior. Most macrophages in cancer are derived from haemopoietic lineages and are continuously recycled with a lifespan within the cancer of about two weeks. Developing an effective particle which contains the drug allows function of the macrophage, and subsequent drug release has been challenging, particularly for protein drugs such as cytokines. Despite ongoing research there has been no cell-based delivery system approved for the clinic, although two therapeutics using red blood cells as a drug vehicle are currently in phase III clinical trials $[32,33]$.

Efficient uptake of protein nanoparticles by phagocytes may allow the use of PODS crystals in a Trojan horse strategy to deliver these cytokine depots preferentially or specifically to an area of disease by exploiting the tumor-infiltrating behavior of macrophages [34]. In order for a PODS Trojan horse approach to be viable as a delivery tool for therapeutic proteins, several criteria have to be met. A basic requirement for a drug delivery system is that it does not produce systemic inflammation or cytotoxicity. PODS proteins have already been used to deliver a diverse range of cargo proteins in different animal models and no adverse effects were observed, including in studies of bone remodeling in rats [23] and dogs [35], as well as neuronal and cartilage regeneration in mice (manuscripts in preparation). While there were no signs of increased inflammation detectable in these animal models, which included examinations of distant sites such as the lung, a detailed investigation into interactions between macrophages and PODS and their possible cytotoxic effects remains to be conducted.

Our studies showed that PODS uptake did not impair three attributes required for macrophage tumor infiltration: mobility, chemotaxis, and migration through narrow spaces. Our results demonstrated no effect on overall levels of mobility. The PODS-loaded macrophages could also squeeze through narrow capillary like tubes and migrate towards a chemotactic signal whether that was towards sera or towards chemotactic agents secreted by cancer cells. Therefore, our results indicate that the tumor homing ability of macrophages may not be affected by phagocytosis of PODS or by the embedded IL-2 cargo protein.

The cytokine cargos of PODS-loaded macrophages may be used to shift the tumor microenvironment in several ways. Macrophages can switch between M1 and M2 phenotypes by responding to cues from the local environment. However, this polarization can be transient and is nonbinary with high levels of cell plasticity [36]. In vitro, the secretion of the cytokine IL- 6 is one of many markers used to determine the polarization status of M1 macrophages [37, 38] and was shown to be dependent on particle size, with the highest secretion seen with particles of $0.8 \mu \mathrm{m}$ [37].

FGF-2 has been shown to shift macrophages towards the M2-like phenotype [39]. A shift in polarization could be exploited in cancer therapy. Tumor-associated macrophages (TAMs) usually express an M2 phenotype (alternatively activated subset), which leads to them performing immunosuppressive and tumor-promoting functions. Reprogramming of such cells towards an M1-like phenotype (classically activated subset) may suppress their procancer phenotype and unleash antitumor activity [40, 41]. Unpolarized or M1 polarized macrophages could be loaded with proinflammatory and M1 polarizing stimuli, such as TNF- $\alpha$, IFN- $\gamma$, or IL-6 to support a proinflammatory environment at the tumor site. This could prevent the formation of TAMs and possibly reprogram macrophages already associated with the tumor.

Cytokines which reprogram the activity of other immune cells could also be used as a cargo. Cytokines are key regulators of immune cell activity, and there are many potential targets and mechanisms with the tumor microenvironment which could be addressed. One obvious candidate is IL-2 which is approved for the treatment of metastatic renal cell cancer and metastatic melanoma [42]. The use of high doses is required to achieve efficacy which result in high levels of 
toxicity requiring hospitalization during therapy. In particular, IL-2 causes leaky vasculature [43]. Targeting IL-2 to tumors using macrophages would shift the therapeutic window to increase efficacy while reducing toxicity and allow IL-2 therapy to become more widely used.

This study lays the foundation for the development of PODS for use in phagocytic cell research in general and macrophages in particular. It also provides a proof-of-concept for a PODS-based macrophage-mediated Trojan horse strategy for delivering proteins including cytokines to combat cancer and other diseases.

\section{Data Availability}

All raw data used to support the findings presented in this study are available from the corresponding author upon request.

\section{Conflicts of Interest}

All authors are employees of Cell Guidance Systems the company commercializing the PODS technology. They are also inventors on patents describing the use of PODS for disease therapy.

\section{Authors' Contributions}

AW and NJ collected and assembled the data. AW, CP, and MJ designed the study, analyzed and interpreted the data, and prepared the manuscript. All authors provided final approval of the article prior to submission.

\section{Acknowledgments}

The authors would like to thank Dr. Ciara Whitty and Dr. Raj Gandhi for early observations and initial characterizations of interactions of PODS with nonprofessional phagocytes. Furthermore, we would like to thank Prof Ann Ager for helpful discussions and comments on the manuscript.

\section{Supplementary Materials}

Supplementary 1. Supp fig 1: PODS morphology. PODS Empty was spun down onto a tissue culture plastic and imaged using SEM (left image) and brightfield microscopy (right image) at a magnification of 40x. PODS are easily distinguishable from cells due to their cubic shape.

Supplementary 2. Supp video 1: mobility of BMDMs after PODS GM-CSF uptake. BMDMs loaded with an average of 5 PODS GM-CSF per cell were monitored with a life cell imaging system for $24 \mathrm{~h}$. This video shows $52 \mathrm{~s}$ equivalent to approximately $4 \mathrm{~h}$ and $20 \mathrm{~min}$ in real time.

Supplementary 3. Supp fig 2: GM-CSF-loaded mouse BMDMs were incubated with PODS eGFP for $24 \mathrm{~h}$ and monitored using a live cell imaging system at $1 \mathrm{frame} / \mathrm{min}$. The dashed white circle follows the movement of a macrophage towards a single PODS, the uptake of the PODS, and the contraction of the macrophage over 21 min depicted in a still of 4 frames $(59,70,76$, and 80$)$.
Supplementary 4. Supp video 2: uptake of PODS eGFP into PODS GM-CSF-loaded BMDMs. BMDMs loaded with an average of 5 PODS GM-CSF per cell were incubated with PODS eGFP at a ratio of 5 PODS eGFP per cell and monitored with a life cell imaging system for $24 \mathrm{~h}$. This video shows $26 \mathrm{~s}$ equivalent to approximately $2 \mathrm{~h}$ and $10 \mathrm{~min}$ in real time.

Supplementary 5. Supp Fig 3: efficient uptake of PODS into M0. THP-1 cells were differentiated first into M0 macrophages and then further polarized into M1 cells. Uptake of PODS Empty was allowed for $24 \mathrm{~h}$ and monitored using a live cell imaging system at 1 frame/min. Three frames $(1,675$, and 1335) are shown to illustrate the uptake over time.

Supplementary 6. Supp Fig 4: phagocytosis of PODS protein crystals into nonprofessional phagocytes. Chondrocytes, NIH3T3 cells, and C2C12 cells were cultured with PODS Empty for $24 \mathrm{~h}$ and subsequently imaged with brightfield microscopy at either $20 \mathrm{x}$ or $40 \mathrm{x}$ magnification. The dashed square marks the area of the zoom shown in the right image of each panel.

Supplementary 7. Supp Fig 5: viability of PODS-loaded macrophages after $96 \mathrm{~h}$. M0, M1, and M2 macrophages were incubated with PODS Empty or PODS FGF-10 (1:5 and $1: 15$ ) for $24 \mathrm{~h}$ in a 96-well TC plate. Subsequently, the medium was changed and viability of cells was measured $96 \mathrm{~h}$ after PODS uptake using a colorimetric assay (Orangu ${ }^{\mathrm{TM}}$, Cell Guidance Systems). The fold change in viability was calculated relative to unloaded macrophages of the same polarization state. Error bars represent the standard deviation of samples $(n=3) .{ }^{*}$ Significant differences to control $(p<0.05)$.

Supplementary 8. Supp Fig 6: overloading of macrophages leads to apoptosis. M1 cells already loaded with an average of 10 PODS per cell and were incubated with another 10 PODS per cell for further $24 \mathrm{~h}$, and images were taken every $2 \mathrm{~min}$. Three frames were chosen to follow 3 cells. The dashed white circle marks a healthy cell with approximately 10 PODS ingested. The white arrow and turquois arrow mark two cells with more than 50 PODS ingested; both cells undergo apoptosis shown in frame 190 and frame 690, respectively.

\section{References}

[1] J. J. Lim, S. Grinstein, and Z. Roth, "Diversity and versatility of phagocytosis: roles in innate immunity, tissue remodeling, and homeostasis," Frontiers in Cellular and Infection Microbiology, vol. 7, 2017.

[2] Y. Okabe and R. Medzhitov, "Tissue biology perspective on macrophages," Nature Immunology, vol. 17, no. 1, pp. 9-17, 2016.

[3] K. Kikuchi, M. Iida, N. Ikeda et al., "Macrophages switch their phenotype by regulating Maf expression during different phases of inflammation," Journal of Immunology, vol. 201, no. 2, pp. 635-651, 2018.

[4] M. Gschwandtner, R. Derler, and K. S. Midwood, "More than just attractive: how CCL2 influences myeloid cell behavior beyond chemotaxis," Frontiers in Immunology, vol. 10, 2019. 
[5] H. Yang, Q. Zhang, M. Xu et al., "CCL2-CCR2 axis recruits tumor associated macrophages to induce immune evasion through PD-1 signaling in esophageal carcinogenesis," Molecular Cancer, vol. 19, no. 1, p. 41, 2020.

[6] R. C. Dandekar, A. V. Kingaonkar, and G. S. Dhabekar, "Role of macrophages in malignancy," Annals of Maxillofacial Surgery, vol. 1, no. 2, pp. 150-154, 2011.

[7] A. S. Poltavets, P. A. Vishnyakova, A. V. Elchaninov, G. T. Sukhikh, and T. K. Fatkhudinov, "Macrophage modification strategies for efficient cell therapy," Cell, vol. 9, no. 6, p. 1535, 2020.

[8] C. Wang, K. Li, T. Li et al., "Monocyte-mediated chemotherapy drug delivery in glioblastoma," Nanomedicine, vol. 13, pp. 157-178, 2018.

[9] M. A. Evans, P.-J. Huang, Y. Iwamoto et al., "Macrophagemediated delivery of light activated nitric oxide prodrugs with spatial, temporal and concentration control," Chemical Science, vol. 9, no. 15, pp. 3729-3741, 2018.

[10] F. Xu, A. Bandara, H. Akiyama et al., "Membrane-wrapped nanoparticles probe divergent roles of GM3 and phosphatidylserine in lipid-mediated viral entry pathways," Proceedings of the National Academy of Sciences of the United States of America, vol. 115, no. 39, pp. E9041-E9050, 2018.

[11] M. S. Abdelgawwad, W. Cao, L. Zheng, N. K. Kocher, L. A. Williams, and X. Long Zheng, "Transfusion of platelets loaded with recombinant ADAMTS13 (a disintegrin and metalloprotease with thrombospondin type 1 repeats-13) is efficacious for inhibiting arterial thrombosis associated with thrombotic thrombocytopenic purpura," Arteriosclerosis, Thrombosis, and Vascular Biology, vol. 38, no. 11, pp. 2731-2743, 2018.

[12] L. Chessa, V. Leuzzi, A. Plebani et al., "Intra-erythrocyte infusion of dexamethasone reduces neurological symptoms in ataxia teleangiectasia patients: results of a phase 2 trial," Orphanet Journal of Rare Diseases, vol. 9, no. 1, p. 5, 2014.

[13] J. P. Dutcher, D. J. Schwartzentruber, H. L. Kaufman et al., "High dose interleukin-2 (Aldesleukin) - expert consensus on best management practices-2014," Journal for Immunotherapy of Cancer, vol. 2, no. 1, p. 26, 2014.

[14] F. Coulibaly, E. Chiu, K. Ikeda et al., "The molecular organization of cypovirus polyhedra," Nature, vol. 446, no. 7131, pp. 97-101, 2007.

[15] J. A. Champion, A. Walker, and S. Mitragotri, "Role of particle size in phagocytosis of polymeric microspheres," Pharmaceutical Research, vol. 25, no. 8, pp. 1815-1821, 2008.

[16] M. Wagner, H. Koester, C. Deffge et al., "Isolation and intravenous injection of murine bone marrow derived monocytes," Journal of Visualized Experiments, vol. 94, no. article e52347, 2014.

[17] M. Genin, F. Clement, A. Fattaccioli, M. Raes, and C. Michiels, "M1 and M2 macrophages derived from THP-1 cells differentially modulate the response of cancer cells to etoposide," $B M C$ Cancer, vol. 15, no. 1, p. 577, 2015.

[18] S. Lanone, F. Rogerieux, J. Geys et al., "Comparative toxicity of 24 manufactured nanoparticles in human alveolar epithelial and macrophage cell lines," Particle and Fibre Toxicology, vol. 6, no. 1, p. 14, 2009.

[19] M. Akter, M. T. Sikder, M. M. Rahman et al., "A systematic review on silver nanoparticles-induced cytotoxicity: physicochemical properties and perspectives," Journal of Advanced Research, vol. 9, pp. 1-16, 2018.
[20] Q. Feng, Y. Liu, J. Huang, K. Chen, J. Huang, and K. Xiao, "Uptake, distribution, clearance, and toxicity of iron oxide nanoparticles with different sizes and coatings," Scientific Reports, vol. 8, no. 1, article 2082, 2018.

[21] J. Chen, Z. Wang, Z. Zheng et al., "Neuron and microglia/macrophage-derived FGF10 activate neuronal FGFR2/PI3K/Akt signaling and inhibit microglia/macrophages TLR4/NF- $\kappa \mathrm{B}-$ dependent neuroinflammation to improve functional recovery after spinal cord injury," Cell Death \& Disease, vol. 8, no. 10, pp. e3090-e3090, 2017.

[22] N. Nishishita, H. Ijiri, C. Takenaka et al., "The use of leukemia inhibitory factor immobilized on virus-derived polyhedra to support the proliferation of mouse embryonic and induced pluripotent stem cells," Biomaterials, vol. 32, no. 14, pp. 3555-3563, 2011.

[23] G. Matsumoto, T. Ueda, J. Shimoyama et al., "Bone regeneration by polyhedral microcrystals from silkworm virus," Scientific Reports, vol. 2, no. 1, p. 935, 2012.

[24] H. Ijiri, F. Coulibaly, G. Nishimura et al., "Structure-based targeting of bioactive proteins into cypovirus polyhedra and application to immobilized cytokines for mammalian cell culture," Biomaterials, vol. 30, no. 26, pp. 4297-4308, 2009.

[25] J. M. Rios de la Rosa, A. Tirella, A. Gennari, I. J. Stratford, and N. Tirelli, "The CD44-mediated uptake of hyaluronic acidbased carriers in macrophages," Advanced Healthcare Materials, vol. 6, article 1601012, 2017.

[26] D. Paul, S. Achouri, Y.-Z. Yoon, J. Herre, C. E. Bryant, and P. Cicuta, "Phagocytosis dynamics depends on target shape," Biophysical Journal, vol. 105, no. 5, pp. 1143-1150, 2013.

[27] D. M. Richards and R. G. Endres, "The mechanism of phagocytosis: two stages of engulfment," Biophysical Journal, vol. 107, no. 7, pp. 1542-1553, 2014.

[28] H. Mori, "Immobilization of bioactive growth factors into cubic proteinous microcrystals (cypovirus polyhedra) and control of cell proliferation and differentiation," TechConnect Briefs, vol. 3, pp. 222-225, 2010.

[29] I. Kadiu, A. Nowacek, J. McMillan, and H. E. Gendelman, "Macrophage endocytic trafficking of antiretroviral nanoparticles," Nanomedicine, vol. 6, no. 6, pp. 975-994, 2011.

[30] G. M. Ihler, R. H. Glew, and F. W. Schnure, "Enzyme loading of erythrocytes," Proceedings of the National Academy of Sciences of the United States of America, vol. 70, no. 9, pp. 2663-2666, 1973.

[31] W. A. Cafruny and S. E. Bradley, "Trojan horse macrophages: studies with the murine lactate dehydrogenase-elevating virus and implications for sexually transmitted virus infection," The Journal of General Virology, vol. 77, no. 12, pp. 3005-3012, 1996.

[32] Erydel, Multi-center, Randomized, Double-blind, Placebocontrolled Trial to Evaluate the Effects of Intra-Erythrocyte Dexamethasone Sodium Phosphate on Neurological Symptoms in Patients With Ataxia Telangiectasia, clinicaltrials.gov, 2020, October 2020, https://clinicaltrials.gov/ct2/show/ NCT02770807.

[33] ERYtech Pharma, A Randomized, Phase 3 Study of Eryaspase in Combination With Chemotherapy Versus Chemotherapy Alone as 2nd-Line Treatment of Patients With Pancreatic Adenocarcinoma, clinicaltrials.gov, 2020, October 2020, https:// clinicaltrials.gov/ct2/show/NCT03665441. 
[34] Y. Qi, X. Yan, T. Xia, and S. Liu, "Use of macrophage as a Trojan horse for cancer nanotheranostics," Materials and Design, vol. 198, article 109388, 2021.

[35] G. Matsumoto, T. Ueda, Y. Sugita et al., "Polyhedral microcrystals encapsulating bone morphogenetic protein 2 improve healing in the alveolar ridge," Journal of Biomaterials Applications, vol. 30, no. 2, pp. 193-200, 2015.

[36] S. X. Liu, H. H. Gustafson, D. L. Jackson, S. H. Pun, and C. Trapnell, "Trajectory analysis quantifies transcriptional plasticity during macrophage polarization," Scientific Reports, vol. 10, no. 1, article 12273, 2020.

[37] G. Arango Duque and A. Descoteaux, "Macrophage cytokines: involvement in immunity and infectious diseases," Frontiers in Immunology, vol. 5, 2014.

[38] M. Orecchioni, Y. Ghosheh, A. B. Pramod, and K. Ley, "Macrophage polarization: different gene signatures in M1(LPS+) vs. classically and M2(LPS-) vs. alternatively activated macrophages," Frontiers in Immunology, vol. 10, 2019.

[39] J. H. Im, J. N. Buzzelli, K. Jones et al., "FGF2 alters macrophage polarization, tumour immunity and growth and can be targeted during radiotherapy," Nature Communications, vol. 11, no. 1, article 4064, 2020.

[40] G. Genard, S. Lucas, and C. Michiels, "Reprogramming of tumor-associated macrophages with anticancer therapies: radiotherapy versus chemo- and immunotherapies," Frontiers in Immunology, vol. 8, 2017.

[41] F. Zhang, N. N. Parayath, C. I. Ene et al., "Genetic programming of macrophages to perform anti-tumor functions using targeted mRNA nanocarriers," Nature Communications, vol. 10, no. 1, article 3974, 2019.

[42] J. P. Dutcher, "Current status of interleukin-2 therapy for metastatic renal cell carcinoma and metastatic melanoma," Oncology, vol. 16, no. 11, Supplement 13, pp. 4-10, 2002.

[43] X. Chen, X. Ai, C. Wu et al., "A novel human IL-2 mutein with minimal systemic toxicity exerts greater antitumor efficacy than wild-type IL-2," Cell Death \& Disease, vol. 9, no. 10, p. 989,2018 